\title{
BMJ Open Protocol for a prospective evaluation of postpartum engagement in HIV care among women living with HIV in South Africa
}

\author{
Christina Psaros, ${ }^{1,2}$ Amelia M Stanton, ${ }^{1,2}$ C Andres Bedoya, ${ }^{1,2}$ Nzwakie Mosery, ${ }^{3}$ \\ Shannon Evans, ${ }^{3}$ Lynn Turner Matthews (D) , Jessica Haberer, ${ }^{1,5}$ Mark Vangel, ${ }^{1,6}$ \\ Steven Safren, ${ }^{7}$ Jennifer A Smit ${ }^{3}$
}

To cite: Psaros C, Stanton AM, Bedoya CA, et al. Protocol for a prospective evaluation of postpartum engagement in HIV care among women living with HIV in South Africa. BMJ Open 2020;10:e035465. doi:10.1136/ bmjopen-2019-035465

- Prepublication history for this paper is available online To view these files, please visit the journal online (http://dx.doi org/10.1136/bmjopen-2019035465).

Received 11 November 2019 Revised 27 November 2019 Accepted 28 November 2019

Check for updates

(c) Author(s) (or their employer(s)) 2020. Re-use permitted under CC BY-NC. No commercial re-use. See rights and permissions. Published by BMJ.

For numbered affiliations see end of article.

Correspondence to Dr Christina Psaros; cpsaros@mgh.harvard.edu

\section{ABSTRACT}

Introduction KwaZulu-Natal (KZN), South Africa (SA) has the highest prevalence of pregnant women living with HIV in the world. Pregnancy and the postpartum period offer opportunities to engage women in HIV care, to prevent perinatal transmission and to optimise maternal and infant well-being. However, research suggests that remaining engaged in HIV care during this time can be challenging. Methods and analysis We are conducting a 5-year prospective cohort study among pregnant women living with HIV in KZN to estimate the rates and factors associated with attrition from HIV care during this critical period. To determine who is most likely to fall out of care, we are examining a range of relevant variables informed by a socioecological model of HIV care, including individual, relational, community and healthcare system variables. We are enrolling 18-45-year-old women, at 28 weeks or more of pregnancy, who are living with HIV and currently taking antiretroviral therapies. Participants complete quantitative assessments at baseline (pregnancy) and at 6, 12, 18 and 24 months postpartum. A subset of women and their partners are invited to complete qualitative interviews to further explore their experiences in HIV care. The main study outcomes are suppressed HIV RNA and retention in care at each study assessment. Our understanding of the factors that drive postpartum attrition from HIV care will ultimately inform the development of interventions to facilitate continued engagement in postpartum HIV care.

Ethics and dissemination This protocol has been approved by the Human Research Ethics Committee (Medical) at The University of the Witwatersrand (Johannesburg, SA) and the Partners Human Research Committee at Partners HealthCare (Boston, Massachusetts, USA). Site support and approval were obtained from the District Hospital and the KZN Provincial Department of Health. Results will be disseminated through peerreviewed manuscripts, reports and both local and international presentations (Ethics Registration \#170212).

\section{INTRODUCTION}

South Africa bears a substantial HIV disease burden, with an estimated 7.9 million people living with HIV in 2017. In the Province
Strengths and limitations of this study

- This study is one of few longitudinal assessments of postpartum engagement in HIV care among women in KwaZulu-Natal and among the first since the South African antiretroviral guidelines changed in 2016, offering lifetime treatment for all persons living with HIV.

- This study tests a multilevel model of HIV care that includes an assessment of facilitators and barriers of engagement in care.

- By employing robust, multilevel tracing methods, this study may be able distinguish participants who move their care elsewhere versus are truly lost to care, thus more accurately estimating attrition.

- We only recruit women engaged in antenatal care. It is likely that pregnant women who do not initiate antenatal care at all are different from the women in our sample.

- Unsuppressed HIV RNA may fail to capture the experiences of women who remain engaged in HIV care but elect to stop treatment or those who have drug-resistant virus. However, we are collecting other data (eg, retention, drug resistance testing) that will provide important information on these women.

of KwaZulu-Natal (KZN), the populationweighted HIV prevalence in 2014-2015 was $36 \%$ overall, $44 \%$ in women and $28 \%$ in men. ${ }^{2} \mathrm{KZN}$ has the highest prevalence of HIV among pregnant women in the world. ${ }^{3}$ Data from South African antenatal clinics suggest elevated rates of HIV infection among pregnant women, with a 2015 national survey documenting rates as high as $46 \%$ among women presenting for antenatal care. ${ }^{3-8}$ Approximately $20 \%$ of pregnant women in South Africa do not receive any HIV treatment, and an estimated 21000 children are infected with HIV each year. ${ }^{9}$

Pregnancy and the postpartum periods present important opportunities to engage 
women in HIV care, to prevent mother-to-child transmission (PMTCT) and to optimise maternal child health. As of 1 September 2016, universal test and treat guidelines articulate that all persons living with HIV in South Africa are eligible for life-long antiretroviral (ARV) treatment, regardless of $\mathrm{CD} 4$ count; pregnant and breastfeeding women living with HIV are considered to be of 'immediate priority'. ${ }^{10}{ }^{11}$ The PMTCT 'cascade' consists of a series of effective but complex steps to which women must adhere to minimise risk of perinatal HIV transmission. These steps, which include the administration of highly active ARV therapy to the mother and ARV prophylaxis to the infant, help prevent HIV infections from a mother living with HIV to her child during pregnancy, labour or delivery and breastfeeding. Adherence to PMTCT during pregnancy has been widely studied; risk factors for nonadherence include lack of partner support around HIV status, fear of disclosure of HIV status and stigma, gender inequality, structural barriers such as geographic distance from clinic and poverty. ${ }^{12-16}$ With PMTCT interventions, the rate of perinatal HIV transmission is approximately $1.4 \%-5.9 \%$, averaging $3.5 \%{ }^{17}$; however, the aforementioned barriers to adherence must be addressed to achieve these levels.

The postpartum period differs from pregnancy in ways that may present barriers to continued HIV care engagement. ${ }^{18}$ After pregnancy, women are asked to administer antiretroviral drugs (ARVs) to their infants, to have their infants tested for HIV and to adhere to breastfeeding recommendations. The WHO recommends that mothers living with HIV should exclusively breastfeed their infants for the first 6 months and may continue breastfeeding for up to 24 months or longer while being supported for antiretroviral therapy (ART) adherence. ${ }^{19}$ In some cases, worry around delivering a healthy infant subsides, leading to changes in motivation for remaining on HIV treatment or in care. ${ }^{20}$ Stigma ${ }^{671321-24}$ and structural barriers, ${ }^{8}$ such as transferring care to another clinic after delivery, are not uncommon. The demands of having a newborn, high rates of depressive symptoms ${ }^{325}$ and need to return to work pose additional barriers. The conclusion of formal PMTCT programming is a 'cliff' in the HIV treatment cascade over which women can fall. ${ }^{26}$

Research has examined attrition from HIV care during the postpartum period, but few studies have documented adherence to ARVs beyond 6-12 months postpartum. ${ }^{11} 27-30$ Existing data for women living with HIV show reduced retention in care during the postpartum period. A systematic review of studies from the USA, Kenya, Zambia and South Africa revealed that ART adherence levels decreased significantly from $74 \%$ during pregnancy to $50 \%$ postpartum. ${ }^{31}$ Data from the PROMISE study, a randomised trial conducted in seven countries to compare the efficacy of prolonged infant ARVs versus maternal ART for PMTCT throughout the breastfeeding period, revealed a virological failure rate of $23 \%$ among women randomised to continued postpartum ARV use. ${ }^{32}$ Notably, the PROMISE study included data from only one African country (Botswana) and non-adherence is likely to be worse among communitybased patients as opposed to those participating in clinical trials. Results from a study that examined the effects of integrating HIV care into antenatal clinics in Kenya also revealed high attrition rates. Only $28 \%$ of women followed up at their antenatal clinics for HIV care postpartum, suggesting additional barriers beyond service integration. ${ }^{33}$ Similarly, in a Ugandan study, only $38 \%$ of mothers enrolled in a postnatal PMTCT programme adhered to a 6 week visit (as measured by attendance at their scheduled appointment). ${ }^{24}$ A prospective study of pregnant women living with HIV in Tanzania showed that $25 \%$ and $41 \%$ of participants at 12 and 24 months postpartum, respectively, met criteria for immunological failure, indicating a direct association between disease progression and months since childbirth. ${ }^{28}$ Retention rates are comparable in South Africa. A study conducted in Cape Town found significant attrition from HIV care after delivery, with only $31 \%$ of the sample continuously reporting engagement in HIV care at 6, 18 and 36 months postpartum. ${ }^{34}$ Young women and women who moved to a variety of clinics for services were particularly vulnerable to attrition. ${ }^{35}$ These data demonstrate that long-term retention in HIV care during the postpartum period remains a challenge.

Without continued engagement in care, women living with HIV and their children risk compromised health outcomes. Women who are not retained in care may not complete infant-specific PMTCT procedures, thus increasing the risk of perinatal transmission. ${ }^{27}$ Individuals who repeatedly start and stop HIV treatment are at risk for HIV viraemia/virological failure, CD4 decline, drug resistance and disease progression to AIDS or death. ${ }^{59}{ }^{29-38}$ Should women become pregnant again, those off treatment may not be in optimal health, and the likelihood of perinatal transmission is higher.

This manuscript describes a longitudinal prospective study that examines the scope of attrition from HIV care following childbirth. As one of the first longitudinal studies of postpartum engagement in HIV care and the first among women in KZN, this project will inform both our understanding of the trajectory of maintenance in HIV care and potential intervention development for this important population.

\section{Study aims and outcomes}

The primary aims of this study are to: (1) estimate the rate of attrition from HIV care during the postpartum period and (2) identify and explore factors associated with attrition from HIV care and failure to achieve viral suppression, during the postpartum period. The primary quantitative outcomes will be HIV RNA (ie, viral suppression) and self-reported number of visits to any HIV care provider (ie, retention). We will also use qualitative methods to further explore barriers and facilitators to care among a subset of participants and their partners. 


\section{METHODS}

\section{Study design}

This is a prospective cohort study of women enrolled in a PMTCT programme, planned to be conducted between 9 June 2017 and 31 May 2022. Up to 500 HIV-positive women between the ages of 18 and 45 will be recruited. The study includes five assessment points: baseline (pregnancy) and 6, 12, 18 and 24 months postpartum. At each assessment, participants complete a quantitative interview and blood draws to measure HIV RNA, which is stored for HIV genotyping, if appropriate. Stored blood samples will be discarded at the end of the study.

The baseline assessment occurs on the same day that a participant consents to study participation, unless she prefers that the visit occur on another day (eg, to give her the chance to discuss participation with her partner and/ or family). Data collected during this assessment include individual (eg, health beliefs, depression, substance use), relational (eg, social support, relationships with intimate partners, intimate partner violence, HIV disclosure), community (eg, poverty, stigma, work commitment) and healthcare system (eg, knowledge of service integration, HIV clinic distance, time waiting, time travelling to clinic) constructs. Medical details are also assessed related to HIV (eg, CD4 count, current medications) as well as pregnancy (eg, antenatal visits attended) and postpartum (eg, infant weight, gestational age, HIV status) periods. Please see table 1 for details.

Optional qualitative interviews are planned to occur during the follow-up period among a subsample of female participants $(\mathrm{n} \sim 50)$. These interviews will be conducted among three groups of women: those who achieved suppressed HIV RNA for at least two consecutive assessments (ie, sustained suppression; $\mathrm{n}=12-15$, depending on thematic saturation), those who did not achieve suppressed HIV RNA for at least two consecutive assessments (ie, sustained non-suppression; $\mathrm{n}=12-15$ ) and those who moved from suppressed HIV RNA to unsuppressed HIV RNA or the reverse $(n=14-20)$. To obtain a diversity of experiences, a proportion of participants meeting these criteria will be offered the qualitative interview after their major assessment visit (ie, 6, 12 and 18 month visits). We will also interview male partners for a subsample of these women (up to 25); women must invite their male partners to participate to avoid inadvertent disclosure of HIV status.

To estimate true attrition from care, it is important to determine if a participant who is seemingly lost to follow-up is simply receiving care elsewhere. We employ a number of tracing techniques to keep track of participants throughout the course of the study if a participant cannot be contacted by phone or does not attend a scheduled visit; participants have the option of consenting to each of these strategies. First, we seek permission to contact participants via social media (eg, Facebook, WhatsApp). We also collect the names of up to 10 individuals who might know how to connect with the participant and reach out to these individuals if the
Table 1 Measures included at each major assessment

$\begin{array}{lllll}B L & 6 M & 12 M & 18 M & 24 M\end{array}$

Sociodemographics (BL)

Sociodemographics (Follow-up)

Sociodemographics (Partner)

Medical: Clinical (BL)

Medical: Clinical (Follow-up)

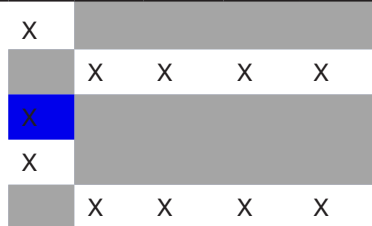

Fertility Desire: Pregnancy Risk Assessment $\mathrm{X}$ Monitoring System (PRAMS) ${ }^{70} 71$

Relational: Partner Characteristics \& Disclosure (BL)

Relational: Partner Characteristics \& Disclosure (Follow-up)

Depression: Centre for Epidemiological Studies Depression - CES-D ${ }^{72}$

Coping: Brief Cope ${ }^{58}$

Resilience: Connor Davidson Resilience Scale - CD-RISC ${ }^{73}$

Substance Use: ASI Lite ${ }^{56}$

Health Beliefs: Perceived HIV Management $^{74}$

Adherence: Antiretroviral Adherence (BL) ${ }^{75}$

Adherence: Antiretroviral Adherence (Follow-up) ${ }^{75}$

Intimate Partner Violence: Abuse Assessment Screen

Medical: Infant Outcomes

Delivery Adherence: Adherence to Labour \& Delivery Practices

Paediatric Adherence: Paediatric International Adherence Questionnaire Behaviour Identification (PACTG) ${ }^{76}$

Medical: Breastfeeding

Medical: Family Planning

Stigma: HIV Stigma Scale ${ }^{77}$

Social Support: Modified Duke-UNC Functional Social Support Questionnaire 7879

Healthcare System: Provider Network \& Knowledge of Healthcare System (BL)

Healthcare System: Provider Network \& Knowledge of Healthcare System (Followup)

Healthcare System: Healthcare Relationship $\quad \mathrm{X} \quad \mathrm{X} \quad \mathrm{X} \quad \mathrm{X} \quad \mathrm{X}$ Trust Scale ${ }^{80}$

Sexual Communication: Sexual Communication Scale ${ }^{81}$

Blood Samples (HIV RNA and genotype*)
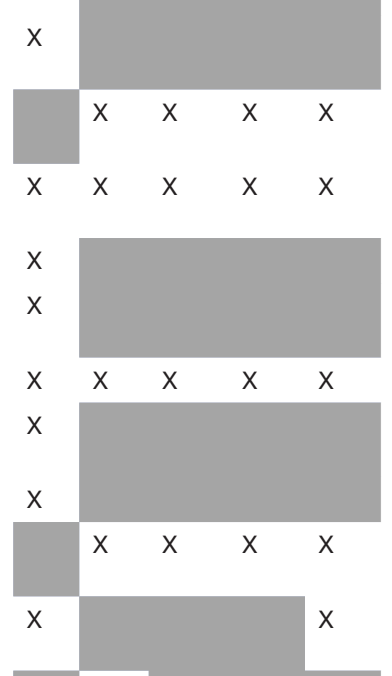

HIV RNA levels are retained at each assessment. Genotype results are retained at 6 months, 12 months, 18 months and 24 months postpartum.

*HIV RNA is entered after study visit.

†Genotyping only done if participants' VL $>1000$

ASI, Addiction Severity Index; BL, baseline; CES-D, Center for Epidemiologica Studies Depression; PACTG, Pediatric AIDS Clinical Trials Group - Pediatric International Adherence Questionnaire Behavior/ldentification; PRAMS, Pregnancy Risk Assessment Monitoring System; VL, viral load.

participant cannot be contacted. During these calls, study staff protect participants' confidentiality by identifying their affiliation with a 'maternal health study' (or some other programme prespecified by the participant). Finally, we have the option to visit the participant's home to conduct the assessment and complete the blood draw. For each missed appointment, study staff can attempt up 


\section{Box 1 Inclusion and exclusion criteria}

\section{Inclusion criteria}

- Living with HIV.

- Aged between 18 and 45 years.

- At 28 weeks of pregnancy or greater (ie, third trimester).

- Currently on antiretroviral therapy.

- Fluent in English or isizulu.

- Access to a phone and willing to give researchers permission to contact them for repeated assessments.

- Able to give informed consent.

\section{Exclusion criteria}

- Active or untreated major mental illness that would interfere with participation (eg, untreated psychosis, bipolar disorder, dementia or active suicidality).

- Participants may be excluded if participation in the study would be unsafe, would complicate the interpretation of study findings or otherwise interfere with study objectives.

to three home visits. If a participant cannot be located to complete an assessment, a review of the participant's medical record is conducted. All data drawn from the National Health Laboratory Service medical record (eg, CD4, HIV RNA) are recorded on a record review form.

\section{Population}

Participants in this study are pregnant women living with HIV who are currently enrolled in a programme to PMTCT of HIV at an antenatal clinic. Women are recruited from a district hospital in a large urban centre in eThekwini District, KZN over a period of 2 years. Although adherence to PMTCT programmes is suboptimal, ${ }^{39-44} 97 \%$ of women in South Africa receive at least some antenatal care. ${ }^{45}$ Participants are recruited during the third trimester of pregnancy (as women in South Africa often present late for antenatal care) ${ }^{46}$

\section{Eligibility criteria}

Study inclusion criteria are presented in box 1 and consist of living with HIV; age between 18 and 45 years; at 28 weeks of pregnancy or greater (ie, in the third trimester); currently on ARV therapy; fluent in English or isiZulu; access to a phone and willing to give researchers permission to contact them for repeated assessments and able to give informed consent.

After initial consent for screening is obtained, participants are assessed for eligibility using a screening script. Participants who meet all criteria and would like to participate sign a second informed consent document to enrol and then complete a full baseline assessment. There are no exclusion criteria with respect to parity or gravity; all participants are eligible for lifetime ARVs per South African HIV treatment guidelines. ${ }^{104748}$ The ARV treatment guidelines also indicate that participants who experience a pregnancy loss are still eligible for lifetime treatment; thus, pregnancy loss will not be exclusionary once enrolled in the study. Women with active or untreated major mental illness that would interfere with participation (eg, untreated psychosis, bipolar disorder, dementia or active suicidality) are excluded and referred for mental health treatment via counsellors at the recruitment site. Participants who are acutely distressed or suicidal will be escorted to on-site mental health services immediately. Potential participants may be excluded if, in the opinion of the Principal Investigator, participation in the study would be unsafe, would complicate interpretation of study findings or otherwise interfere with achieving study objectives.

\section{Individual, relational, community and health system variables}

A range of individual, relational, community and healthcare system variables are measured at each assessment informed by a socioecological model ${ }^{42}$ (figure 1 ). Some of the individual-level variables include substance use, health beliefs and depression. Substance use is assessed with the Addiction Severity Index-Lite, ${ }^{49}$ which probes lifetime and recent substance use, including severity. Health beliefs are measured with the 8-item Perceived HIV Self-Management Scale,$^{50}$ and depression is assessed with the Center for Epidemiologic Studies Depression scale, ${ }^{51}{ }^{52}$ which is widely used in South Africa ${ }^{53}$ and has been validated among pregnant women living with HIV $^{54}$ Examples of relational variables measured throughout the study include social support, intimate partner violence and HIV disclosure. Social support is measured via the Modified Duke-UNC Functional Social Support Questionnaire, ${ }^{556}$ which assesses the availability of emotional, informational and tangible support as well as the number of individuals providing support. The presence of intimate partner violence within the last year is documented with the Abuse Assessment Screen. ${ }^{57} 58$ If the participant answers 'yes' to one or more questions, a clinical assessment is conducted. To assess HIV disclosure, participants are asked to whom they have disclosed their HIV status and if their pregnancy partner is aware of their HIV status. Community-level variables include stigma and employment policies. Stigma is measured with the HIV Stigma Scale ${ }^{59}$ which assesses several domains, such as personalised stigma, disclosure concerns, negative self-image and concerns with public attitudes towards HIV. For participants who report employment, we assess maternity leave and sick time policies. Healthcare system variables include knowledge of service integration (ie, participants' knowledge of how to transfer care from PMTCT to mainstream HIV services after delivery) and specific clinic factors (eg, modes of transportation used to travel to clinic, travel time, amount of time spent at clinic and so on). Other measures that are assessed during the study are included in table 1 .

\section{Study activities}

Quantitative interviews

To maximise the integrity of self-report data, a racially and ethnically concordant female research assistant (whenever possible), fluent in both English and isiZulu, conducts the assessments. The baseline assessment is 


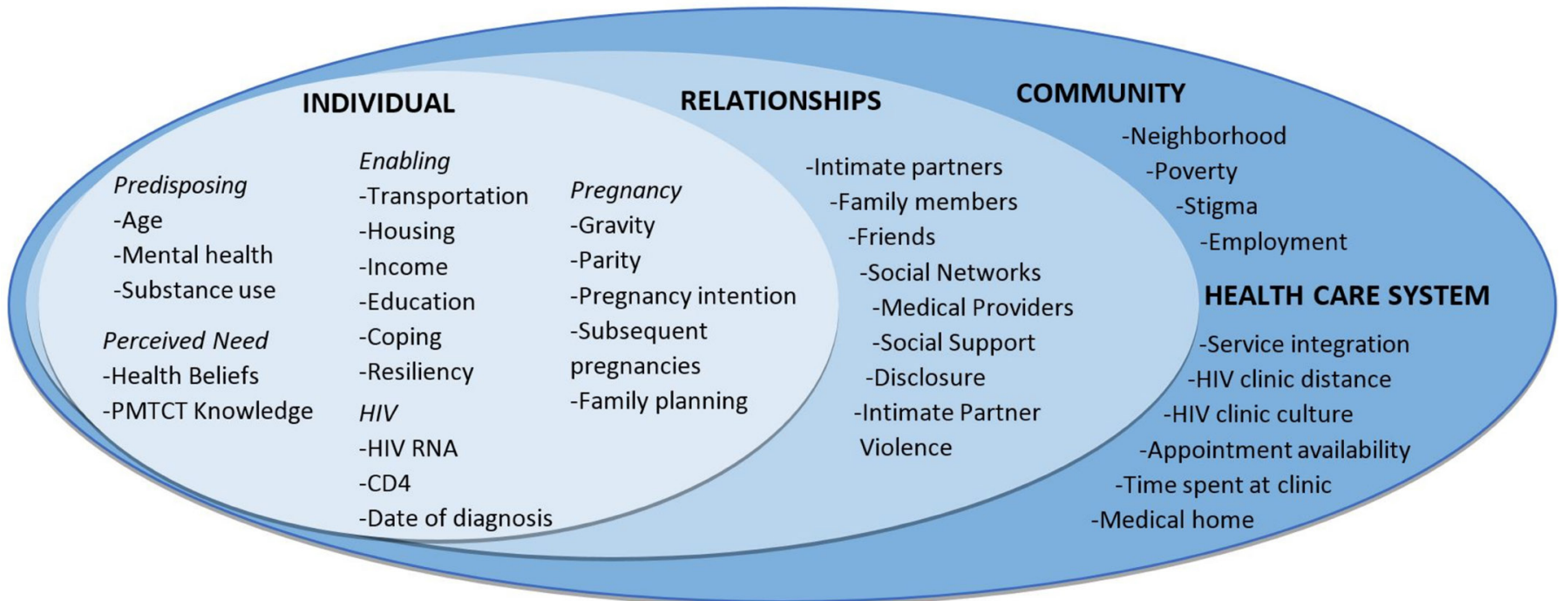

Figure 1 Social-ecological model of factors impacting HIV Care. The study is informed by a social-ecological framework ${ }^{42}$ that has been adapted to address factors potentially relevant to postpartum engagement in HIV care. Study assessments measure these factors at the individual, relational, community and healthcare system levels. PMTCT, prevent mother-to-child transmission.

administered by a trained study staff member using an electronic tablet and takes place in a private room at the recruitment site. The data collected at baseline include basic sociodemographic information (eg, age, educational level and ethnicity) as well as the individual, relationship, community and healthcare system variables described above. Measures that are not readily available in isiZulu have been translated and back-translated and piloted before use. Please see table 1 for an outline of all measures that will be included at each assessment.

\section{Blood collection for HIV RNA}

At all assessment visits, nurses collect two $6 \mathrm{~mL}$ tubes of blood to measure HIV RNA levels, for possible HIV genotyping and/or sample storage. The HIV RNA testing is being conducted at each time point. Whole blood specimens are separated into plasma using standard centrifugation techniques, and HIV RNA is determined via RNA extraction and PCR amplification (this testing is being conducted by Global Clinical and Viral Laboratory (South Africa) using the Biomerieux assay). Participants with unsuppressed HIV RNA are given a letter referring them to their healthcare providers for further care.

\section{HIV genotyping and sample storage}

Although we use detectable HIV RNA as one indicator of attrition from HIV care, viraemia may also occur as a result of ARV resistance. Therefore, we are conducting HIV drug resistance testing for any participant with unsuppressed HIV RNA at the 6-month assessment. If drug resistance is detected, this information is shared with the participant and her identified provider to optimise clinical care. At all other assessments, plasma is stored if HIV RNA is $>1000$ copies/mL to enable more extensive resistance testing should our data warrant it. Only blood samples with HIV RNA $>1000$ copies/mL are retained in storage. Any stored blood samples will be appropriately discarded at the end of the study.

\section{Qualitative interviews}

The participants who are selected to complete the qualitative interview will be categorised according to the three viral suppression patterns described earlier. Participants who are selected for this phase complete up to two interviews between the 6 and 24 months postpartum visits. The male partners complete one interview during this time.

All interview questions are open-ended to most effectively elicit information. For the female participants, the interview includes a free-list exercise to solicit unbiased accounts of experiences with postpartum engagement in HIV care. The participant is asked to list the top five barriers and facilitators of postpartum HIV care. The research assistant then probes to query the topic areas generated by the free-list activity to facilitate depth of discussion. Probes are designed to assess and understand how these factors specifically contributed to success or failure to stay engaged in HIV care, and how interventions may make use of facilitators to support continued engagement in care and/or to overcome barriers to retention in care. In addition to targeting topics generated by the free-list activity, the interviewer asks the participant about her current relationships (family, friends, community, pregnancy partner), perceptions of engagement in postpartum HIV care and how future interventions may support better engagement in postpartum HIV care. During the second interview, the participant is asked about changes in her level of engagement in care, facilitators and barriers experienced since her first interview. 
For the male pregnancy partners, the interview focuses on how partnership characteristics shape attrition from and retention in HIV care during the postpartum period. Interview themes include: his perception of his partner's overall HIV care engagement since pregnancy, ways the romantic relationship impacts partner's HIV care engagement, perceived barriers/facilitators to partner's HIV care engagement and ways to better integrate male pregnancy partners in postpartum HIV care.

\section{Patient and public involvement}

Participants, community members and other relevant stakeholders will be involved in the reporting and dissemination of study findings as appropriate. The study team plans to provide regular (approximately quarterly) updates on the data collection process and study findings to the Community Advisory Board (CAB) as well as an annual update to KZN's Department of Health.

\section{DATA ANALYSIS}

We will estimate the rate of attrition from HIV care and identify factors associated with attrition from care during the postpartum period based on the above-described, comprehensive socioecological model. We will also predict who is most likely to experience postpartum attrition from HIV care using a risk score based on the variables in our socioecological model and our previous work. ${ }^{60}$ We will develop a predictive model for postpartum attrition from HIV care (or detectable HIV RNA), including baseline constructs as covariates, using logistic regression. Following the approach of Steyerberg and colleagues, ${ }^{61}$ we will assess model performance with respect to calibration and discrimination. ${ }^{62}$ Calibration, which refers to the agreement of observed outcome to predicted probabilities, will be assessed using the Hosmer-Lameshow goodness-of-fit test. ${ }^{63}$ Discrimination, the ability to distinguish cases of attrition, will be measured using the area under the receiver operating curve. ${ }^{64}$ The model will be internally validated using the bootstrap technique. ${ }^{65} \mathrm{~A}$ risk score will be determined from the coefficients of the logistic regression model. As additional longitudinal data become available, we will also test to see if these high-risk participants have later unsuppressed HIV viral load more frequently than low-risk participants. We will perform data analyses in SPSS and R.

For the first set of analyses, we will use proportional hazards regression models to estimate the rate of attrition from HIV care, examining HIV RNA as a potential predictor and including selected baseline characteristics as covariates. We will select baseline characteristics based on the literature and clinical judgement, and we will consider them for the predictive model using cross-validated stepwise logistic regression. While some newer studies show resistance testing can be conducted with HIV RNA of $<1000$ copies $/ \mathrm{mL},{ }^{66-68}$ standard assays still generally require HIV RNA >1000 copies/mL. Though we considered censoring participants who had evidence of ARV resistance at the 6-month assessment (to allow time for a change in regimen before the next assessment), we determined that this may result in miscategorising participants who have resistance but cannot be tested for it due to low level viraemia and/or participants who cannot change regimens or achieve suppressed HIV RNA in time for the next assessment, but are still retained in care. Thus, in regression models for HIV RNA, we will likely include indicator variables for whether resistance testing was performed, and whether drug resistance was observed. We will also consider models with visit constancy as the measure of attrition from HIV care, where visit constancy is an ordered categorical variable with levels full retention, partial retention and failure corresponding to at least $80 \%$ of visits, $30 \%-80 \%$ of visits and $<30 \%$ of visits, respectively. ${ }^{69}$ We will employ ordinal regression models to analyse the visit constancy data.

Despite our intensive tracing methods, some participants will ultimately be lost to study procedures. We will examine missing data patterns to see if missingness is related to outcome, and we will employ multiple imputation if the data appear to be missing at random. Of particular importance is missing HIV viral load values for participants lost to follow-up. We expect that a high proportion of these participants will have unsuppressed HIV RNA. To test this hypothesis, we will use two-sample t-tests to compare the HIV RNA of participants who initially miss a study visit, but for whom HIV RNA is later obtained after engaging the intensive tracking methods described above, with the HIV RNA of participants who did not miss a study visit.

Qualitative interviews will be audiotaped, transcribed and translated. Content analyses will be conducted (facilitated by NVivo software) to uncover themes related to postpartum engagement in HIV care. In an iterative multistep process, major themes will be identified first. Then data will be structured into categories to create groups. Themes will be re-examined, and major and minor themes will be identified within each relevant content area. Two coders will analyse the data independently to ensure reliability, and results and discrepancies will be compared and discussed until the coders reach a resolution. An audit trail of coding templates and discussions will be kept to resolve potential discrepancies and to compare computerised coding to the raw interview data.

\section{ETHICS AND DISSEMINATION}

Ethics approval was obtained for all aspects of this study by the Human Research Ethics Committee (Medical) at The University of the Witwatersrand in South Africa, where the work is being conducted, as well as by the Partners Human Research Committee in the USA, where the study was conceived and developed. Study updates and final findings will be communicated to the staff and management of the facility in which the study is being 
conducted and an annual report will be submitted to the KZN Provincial Department of Health throughout the course of the study. Study updates and findings will be shared at the $\mathrm{CAB}$ meetings convened quarterly at Match Research Unit (MRU) and at MRU biannual dissemination days. The data will be also presented at national and international scientific meetings and published in peerreviewed journals.

\section{Author affiliations}

${ }^{1}$ Harvard Medical School, Boston, Massachusetts, USA

${ }^{2}$ Department of Psychiatry, Massachusetts General Hospital, Boston, Massachusetts, USA

${ }^{3}$ MatCH Research Unit (MRU), Department of Obstetrics and Gynaecology, Faculty of Health Sciences, University of Witwatersrand, Durban, South Africa

${ }^{4}$ Department of Medicine, Division of Infectious Diseases, School of Medicine, University of Alabama at Birmingham, Birmingham, Alabama, USA

${ }^{5}$ Center for Global Health, Division of Infectious Diseases, Massachusetts General Hospital, Boston, Massachusetts, USA

${ }^{6}$ Department of Radiology, Massachusetts General Hospital, Boston, Massachusetts, USA

${ }^{7}$ Department of Psychology, University of Miami, Coral Gables, Florida, USA

Contributors $\mathrm{CP}$ is the principal investigator (PI) of the study. AS is a postdoctoral fellow on the project and led the development of the manuscript with $\mathrm{CP}$ and with input from all authors. CAB is the US-based project director coordinating study operations, including managing the database. NM is the South African-based project director; in this capacity, NM helps oversee all study operations, including supervising and training research assistants to collect the data. SE serves as medical director of the project and is therefore responsible for managing the HIV RNA testing, genotyping and sample storage. LTM, JH and SS are consultants with expertise in women's reproductive health and the psychosocial aspects of HIV care. $\mathrm{MV}$ is responsible for conducting the statistical analyses and JAS is the site PI.

Funding This work is supported by funding from the NIH (R01 MH112385-01; 5T32MH116140-02). All authors, except for AS, were funded by R01 MH112385-01 (PI: Psaros). AS is funded by a T32 training grant (5T32MH116140-02). The content is solely the responsibility of the authors and does not necessarily represent the official views of the National Institutes of Health.

Competing interests LTM has worked as a paid consultant for Merck Pharmaceuticals.

Patient consent for publication Not required.

Provenance and peer review Not commissioned; externally reviewed.

Open access This is an open access article distributed in accordance with the Creative Commons Attribution Non Commercial (CC BY-NC 4.0) license, which permits others to distribute, remix, adapt, build upon this work non-commercially, and license their derivative works on different terms, provided the original work is properly cited, appropriate credit is given, any changes made indicated, and the use is non-commercial. See: http://creativecommons.org/licenses/by-nc/4.0/.

\section{ORCID iD}

Lynn Turner Matthews http://orcid.org/0000-0001-6167-6328

\section{REFERENCES}

1 Simbayi L, Zuma K, Moyo S. South African national HIV prevalence, incidence, behaviour and communication survey, 2017, 2018.

2 Kharsany ABM, Cawood C, Khanyile D, et al. Community-Based HIV prevalence in KwaZulu-Natal, South Africa: results of a crosssectional household survey. Lancet HIV 2018;5:e427-37.

3 National Department of Health. The 2013 national antenatal sentinel HIV prevalence survey, South Africa, 2015. Available: http://www. health-e.org.za/wp-content/uploads/2016/03/Dept-Health-HIV-HighRes-7102015.pdf

4 UNAIDS. South Africa: HIV and AIDS estimates Factsheet (2016), 2017. Available: http://www.unaids.org/en/regionscountries/ countries/southafrica
5 Clouse K, Schwartz S, Van Rie A, et al. "What they wanted was to give birth; nothing else": barriers to retention in option B+ HIV care among postpartum women in South Africa. J Acquir Immune Defic Syndr 2014;67:e12-18.

6 The South African National AIDS Council. The National strategic plan. Available: https://sanac.org.za/the-national-strategic-plan/ [Accessed 6 Mar 2019].

7 UNAIDS. Executive summary: how AIDS changed everything MDG6: 15 years, 15 lessons of hope from the AIDS response, 2015. Available: http://www.unaids.org/sites/default/files/media_asset/ MDG6_ExecutiveSummary_en.pdf [Accessed 7 Aug 2017].

8 UNICEF. PMTCT country report, fact sheet, South Africa, 2010. Available: http://www.unicef.org/aids/files/SAfrica_PMTCTFactsheet 2010.pdf [Accessed 28 Feb 2019].

9 Laher F, Cescon A, Lazarus E, et al. Conversations with mothers: exploring reasons for prevention of mother-to-child transmission (PMTCT) failures in the era of programmatic scale-up in Soweto, South Africa. AIDS Behav 2012;16:91-8.

10 World Health Organization. Consolidated guidelines on the use of antiretroviral drugs for treating and preventing HIV infection: recommendations for a public health approach. World Health Organization, 2016.

11 IATT I. Option B+ countries and Pmtct regimen; 2016.

12 Murray LK, Semrau K, McCurley E, et al. Barriers to acceptance and adherence of antiretroviral therapy in urban Zambian women: a qualitative study. AIDS Care 2009;21:78-86.

13 Peltzer K, Ramlagan S. Perceived stigma among patients receiving antiretroviral therapy: a prospective study in KwaZulu-Natal, South Africa. AIDS Care 2011;23:60-8.

14 Sabin LL, Desilva MB, Hamer DH, et al. Barriers to adherence to antiretroviral medications among patients living with HIV in southern China: a qualitative study. AIDS Care 2008;20:1242-50.

15 Simbayi LC, Kalichman S, Strebel A, et al. Internalized stigma, discrimination, and depression among men and women living with HIV/AIDS in Cape town, South Africa. Soc Sci Med 2007:64:1823-31.

16 Phillips T, McNairy ML, Zerbe A, et al. Implementation and operational research: postpartum transfer of care among HIVinfected women initiating antiretroviral therapy during pregnancy. $J$ Acquir Immune Defic Syndr 2015;70:e102-9.

17 Sawyer A, Ayers S, Smith H. Pre- and postnatal psychological wellbeing in Africa: a systematic review. J Affect Disord 2010;123:17-29.

18 Holkmann Olsen C, Mocroft A, Kirk O, et al. Interruption of combination antiretroviral therapy and risk of clinical disease progression to AIDS or death. HIV Med 2007;8:96-104.

19 World Health Organization. Guideline: updates on HIV and infant feeding: the duration of breastfeeding and support from health services to improve feeding practices among mothers living with HIV, 2016.

20 Parienti J-J, Das-Douglas M, Massari V, et al. Not all missed doses are the same: sustained NNRTI treatment interruptions predict HIV rebound at low-to-moderate adherence levels. PLoS One 2008;3:e2783.

21 Cohen MS, Chen YQ, McCauley M, et al. Prevention of HIV1 infection with early antiretroviral therapy. $N$ Engl $J$ Med 2011;365:493-505.

22 Seminari E, De Silvestri A, Boschi A, et al. CD4+ guided antiretroviral treatment interruption in HIV infection: a meta-analysis. AIDS Rev 2008;10:236-44

23 van Lettow M, Bedell R, Landes M, et al. Uptake and outcomes of a prevention-of mother-to-child transmission (PMTCT) program in Zomba district, Malawi. BMC Public Health 2011;11:426.

24 Nassali M, Nakanjako D, Kyabayinze D, et al. Access to HIV/AIDS care for mothers and children in sub-Saharan Africa: adherence to the postnatal PMTCT program. AIDS Care 2009;21:1124-31.

25 South African National Department of Health. Strategic Plan for Maternal, Newborn, Child and Women's Health (MNCWH) and Nutrition in South Africa 2012-2016, 2012.

26 Oyugi JH, Byakika-Tusiime J, Ragland K, et al. Treatment interruptions predict resistance in HIV-positive individuals purchasing fixed-dose combination antiretroviral therapy in Kampala, Uganda. AIDS 2007;21:965-71.

27 Panditrao M, Darak S, Kulkarni V, et al. Socio-demographic factors associated with loss to follow-up of HIV-infected women attending a private sector PMTCT program in Maharashtra, India. AIDS Care 2011;23:593-600.

28 Marcos Y, Phelps BR, Bachman G. Community strategies that improve care and retention along the prevention of mother-to-child transmission of HIV cascade: a review. J Int AIDS Soc 2012;15(Suppl 2):17394. 
29 Rotheram-Borus MJ, Tomlinson M, Scheffler A, et al. Re-engagement in HIV care among mothers living with HIV in South Africa over 36 months post-birth. AIDS 2015;29:2361-2.

30 Rotheram-Borus MJ, Tomlinson M, Scheffler A, et al. Adjustment of a population of South African children of mothers living With/ and without HIV through three years Post-Birth. AIDS Behav 2017:21:1601-10.

31 Nachega JB, Uthman OA, Anderson J, et al. Adherence to antiretroviral therapy during and after pregnancy in low-income, middle-income, and high-income countries: a systematic review and meta-analysis. AIDS 2012;26:2039-52.

32 Currier J, Britto P, Hoffman R. Randomized trial of stopping or continuing ART among postpartum women with pre-ART CD4> 400 cells/mm3 (PROMISE 1077HS). 21st International AIDS Conference, Durban, South Africa, 2016.

33 Washington S, Owuor K, Turan JM, et al. Implementation and operational research: effect of integration of HIV care and treatment into antenatal care clinics on mother-to-child HIV transmission and maternal outcomes in Nyanza, Kenya: results from the SHAIP cluster randomized controlled trial. J Acquir Immune Defic Syndr 2015;69:e164.

34 Moth IA, Ayayo ABCO, Kaseje DO. Assessment of utilisation of PMTCT services at Nyanza provincial Hospital, Kenya. SAHARA-J 2005;2:244-50

35 Phillips TK, Clouse K, Zerbe A, et al. Linkage to care, mobility and retention of HIV-positive postpartum women in antiretroviral therapy services in South Africa. J Int AIDS Soc 2018;21(Suppl 4):e25114.

36 Stringer EM, Sinkala M, Stringer JS, et al. Prevention of motherto-child transmission of HIV in Africa: successes and challenges in scaling-up a nevirapine-based program in Lusaka, Zambia. AIDS 2003;17:1377-82.

37 Lambert JS, Avramovic G, Jackson V, et al. Investigation into the reasons for maternal default from HIV care postpartum: a 3-year retrospective review. AIDS Patient Care STDS 2014;28:1-3.

38 Ngarina M, Kilewo C, Karlsson K, et al. Virologic and immunologic failure, drug resistance and mortality during the first 24 months postpartum among HIV-infected women initiated on antiretroviral therapy for life in the Mitra plus Study, Dar es Salaam, Tanzania. $B M C$ Infect Dis 2015;15:175.

39 Psaros C, Mosery N, Smit JA, et al. PMTCT adherence in pregnant South African women: the role of depression, social support, stigma and structural barriers to care. AIDS Res Hum Retroviruses 2014;30:A61.

40 Psaros C. Contextual risk among pregnant, second generation young women in South Africa. presented at the: association for the social sciences and humanities in HIV. Paris, France, 2013.

41 Geng EH, Bangsberg DR, Musinguzi N, et al. Understanding reasons for and outcomes of patients lost to follow-up in antiretroviral therapy programs in Africa through a sampling-based approach. J Acquir Immune Defic Syndr 2010;53:405-11.

42 Mugavero MJ, Norton WE, Saag MS. Health care system and policy factors influencing engagement in HIV medical care: piecing together the fragments of a fractured health care delivery system. Clin Infect Dis 2011:52(Suppl 2):S238-46.

43 Oladokun RE, Brown B, Osinusi K. Loss to follow-up rate, reasons and associated risk factors among mother-infant pairs in a prevention of mother-to-child transmission programme (PMTCT) in Nigeria: a case control study. Niger J Paediatr 2006;33:79-84.

44 Grant E, Logie D, Masura M, et al. Factors facilitating and challenging access and adherence to antiretroviral therapy in a township in the Zambian Copperbelt: a qualitative study. AIDS Care 2008;20:1155-60.

45 Shisana O, Simbayi LC, Rehle T. South African national HIV prevalence, incidence, behaviour and communication survey, 2008: the health of our children. HSRC Press, 2010. http://hdl.handle.net/ 20.500.11910/4165

46 Manikkam L, Burns JK. Antenatal depression and its risk factors: an urban prevalence study in KwaZulu-Natal. $S$ Afr Med $J$ 2012;102:940-4.

47 World Health Organization. Guideline on when to start antiretroviral therapy and on pre-exposure prophylaxis for HIV. World Health Organization, 2015.

48 World Health Organization. Consolidated guidelines on the use of antiretroviral drugs for treating and preventing HIV infection. Geneva, Switzerland: WHO Library Cataloguing-in-Publication Data, 2013

49 Devries KM, Mak JY, Bacchus LJ, et al. Intimate partner violence and incident depressive symptoms and suicide attempts: a systematic review of longitudinal studies. PLoS Med 2013;10:e1001439.

50 Walsh JC, Mandalia S, Gazzard BG. Responses to a 1 month self-report on adherence to antiretroviral therapy are consistent with electronic data and virological treatment outcome. AIDS 2002;16:269-77.

51 O'Gorman DA, Nyirenda LJ, Theobald SJ. Prevention of mother-tochild transmission of HIV infection: views and perceptions about swallowing nevirapine in rural Lilongwe, Malawi. BMC Public Health 2010;10:354.

52 Shamu S, Abrahams N, Temmerman M, et al. A systematic review of African studies on intimate partner violence against pregnant women: prevalence and risk factors. PLoS One 2011;6:e17591.

53 Kaye DK, Mirembe FM, Bantebya G, et al. Domestic violence during pregnancy and risk of low birthweight and maternal complications: a prospective cohort study at Mulago Hospital, Uganda. Trop Med Int Health 2006;11:1576-84.

54 Siemieniuk RAC, Krentz HB, Miller P, et al. The clinical implications of high rates of intimate partner violence against HIV-positive women. $J$ Acquir Immune Defic Syndr 2013;64:32-8.

55 Mundell JP, Visser MJ, Makin JD, et al. The impact of structured support groups for pregnant South African women recently diagnosed HIV positive. Women Health 2011;51:546-65.

56 McLellan AT, Luborsky L, Woody GE, et al. An improved diagnostic evaluation instrument for substance abuse patients. The addiction severity index. J Nerv Ment Dis 1980;168:26-33.

57 Hobkirk AL, Watt MH, Green KT, et al. Mediators of interpersonal violence and drug addiction severity among methamphetamine users in Cape town, South Africa. Addict Behav 2015;42:167-71.

58 Carver CS. You want to measure coping but your protocol' too long: consider the brief cope. Int J Behav Med 1997;4:92-100.

59 Kotzé M, Visser M, Makin J, et al. The coping strategies used over a two-year period by HIV-positive women who had been diagnosed during pregnancy. AIDS Care 2013;25:695-701.

60 Psaros C, Remmert JE, Bangsberg DR, et al. Adherence to HIV care after pregnancy among women in sub-Saharan Africa: falling off the cliff of the treatment cascade. Curr HIVIAIDS Rep 2015;12:1-5.

61 Steyerberg EW, Neville BA, Koppert LB, et al. Surgical mortality in patients with esophageal cancer: development and validation of a simple risk score. J Clin Oncol 2006;24:4277-84.

62 Harrell FE, Lee KL, Mark DB. Multivariable prognostic models: issues in developing models, evaluating assumptions and adequacy, and measuring and reducing errors. Stat Med 1996;15:361-87.

63 Hosmer DW, Hosmer T, Le Cessie S, et al. A comparison of goodness-of-fit tests for the logistic regression model. Stat Med 1997:16:965-80.

64 Steyerberg EW. Clinical prediction models: a practical approach to development, validation, and updating. Springer Science \& Business Media, 2008

65 Steyerberg EW, Eijkemans MJ, Harrell FE, et al. Prognostic modelling with logistic regression analysis: a comparison of selection and estimation methods in small data sets. Stat Med 2000;19:1059-79.

66 Santoro MM, Fabeni L, Armenia D, et al. Reliability and clinical relevance of the HIV-1 drug resistance test in patients with low viremia levels. Clin Infect Dis 2014;58:1156-64.

67 Gonzalez-Serna A, Min JE, Woods C, et al. Performance of HIV-1 drug resistance testing at low-level viremia and its ability to predict future virologic outcomes and viral evolution in treatment-naive individuals. Clin Infect Dis 2014;58:1165-73.

68 Richman DD. Editorial commentary: extending HIV drug resistance testing to low levels of plasma viremia. Oxford University Press, 2014.

69 Rollins NC, Becquet R, Orne-Gliemann J, et al. Defining and analyzing retention-in-care among pregnant and breastfeeding HIV-infected women: unpacking the data to interpret and improve PMTCT outcomes. J Acquir Immune Defic Syndr 2014;67(Suppl 2):S150-6.

70 Nettleman MD, Chung H, Brewer J, et al. Reasons for unprotected intercourse: analysis of the PRAMS survey. Contraception 2007;75:361-6.

71 Williams L, Morrow B, Shulman H. PRAMS 2002 surveillance report. Atlanta: US Department of Health and Human Services, Centers for Disease Control and Division of Reproductive Health, 2006.

72 Radloff LS. The CES-D scale: a self-report depression scale for research in the general population. Appl Psychol Meas 1977;1:385-401.

73 Campbell-Sills L, Stein MB. Psychometric analysis and refinement of the Connor-davidson resilience scale (CD-RISC): validation of a 10-item measure of resilience. J Trauma Stress 2007;20:1019-28.

74 Wallston KA, Osborn CY, Wagner LJ, et al. The perceived medical condition self-management scale applied to persons with HIV/AIDS. $J$ Health Psychol 2011;16:109-15.

75 Lu M, Safren SA, Skolnik PR, et al. Optimal recall period and response task for self-reported HIV medication adherence. AIDS Behav 2008;12:86-94. 
76 NIAID Pediatric AIDS Clinical Trials Group. Pediatric international adherence questionnaire behavior/identification. QL5030 (P0000)/0000-00 PACTG; 2005

77 Jeyaseelan L, Kumar S, Mohanraj R, et al. Assessing HIV/AIDS stigma in South India: validation and Abridgement of the Berger HIV stigma scale. AIDS Behav 2013;17:434-43.

78 Broadhead WE, Gehlbach SH, de Gruy FV, et al. The Duke-UNC functional social support questionnaire. Measurement of social support in family medicine patients. Med Care 1988;26:709-23.
79 Antelman G, Smith Fawzi MC, Kaaya S, et al. Predictors of HIV-1 serostatus disclosure: a prospective study among HIVinfected pregnant women in Dar ES Salaam, Tanzania. AIDS 2001;15:1865-74.

80 Bova C, Route PS, Fennie K, et al. Measuring patient-provider trust in a primary care population: refinement of the health care relationship trust scale. Res Nurs Health 2012;35:397-408.

81 Choi KH, Catania JA, Dolcini MM. Extramarital sex and HIV risk behavior among US adults: results from the National AIDS behavioral survey. Am J Public Health 1994;84:2003-7. 